\title{
The Design and Evaluation of an Assistive Multimodal Interface
}

\author{
Philip Strain \\ Sonic Arts Research Centre \\ Queens University, Belfast \\ BT7 1NN \\ +44 (0)2890974829 \\ p.strain@qub.ac.uk
}

\begin{abstract}
A requirements capture carried out with thirty blind and visually impaired participants has outlined many issues visually impaired people face when accessing the Web using current assistive technology. One key finding was that spatial information is not conveyed to users. An assistive multimodal interface has been developed that conveys spatial information to users via speech, audio and haptics. Additionally, techniques for evaluating assistive technology with visually impaired participants are discussed.
\end{abstract}

\section{Categories and Subject Descriptors}

H.5.2 User Interfaces: auditory feedback \& haptic I/O; H.5.2. Evaluation/methodology; K.4.2 Social Issues: assistive technologies for persons with disabilities

\section{General Terms}

Design, Human Factors.

\section{Keywords}

Accessibility, audio and haptics, evaluation/methodology

\section{INTRODUCTION}

The Web has the potential to enhance the way visually impaired people lead their daily lives. However, due to limitations in current assistive technology, this potential is not being realized. For instance, it is estimated that the web is three times easier to use for sighted users than for the visually impaired [1]. Various factors have been identified to explain this - for example many web sites have not been designed with accessibility in mind and the linear access model employed by screen readers is constrained by user's short-term memory.

A requirements capture consisting of a questionnaire with 30 respondents, several focus groups and observational studies have highlighted some of the issues visually impaired people face when accessing the Internet [4]. Many participants requested access to spatial information. Screen reader models have been categorized as being linear, conversational,

(c) Philip Strain, 2007

Published by the British Computer Society Volume 2 Proceedings of the 21st BCS HCI Group Conference

HCI 2007, 3-7 September 2007, Lancaster University, UK Devina Ramduny-Ellis \& Dorothy Rachovides (Editors) hierarchical or spatial; however current screen reader technology only employs a linear model.

This research is thus concerned with the design and evaluation of an assistive multimodal interface that mediates spatial information to visually impaired users via non-speech audio, haptics and speech.

\section{DESIGN}

An initial prototype was designed that provides access to Web pages via audio, haptics and speech. Input is provided via a force-feedback mouse. Visually impaired users cannot use a standard mouse, due to a lack of feedback, however it was hypothesized that by augmenting a mouse with haptics and audio, users would be able to gain a sense of position on a page. Haptic effects and auditory icons were associated with links, images and the page border. A "torch effect" was employed that informed users if there was an element in the vicinity of the mouse cursor - the location of the element was conveyed via non-speech audio. An initial evaluation [8] of the system found that users were able to develop a spatial representation of a web page. However the distance moved by the mouse did not correspond to the distance moved by the cursor, users were unaware of their position in the context of the page, and one user stated that additional feedback to provide additional awareness of the position of the cursor would be beneficial.

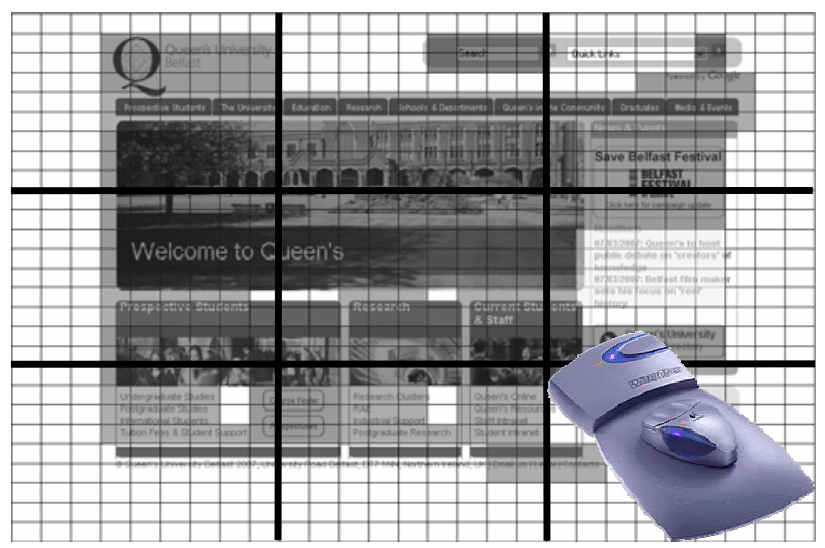

Figure 1 - 27x27 grid with page segmentation

In the second iteration of the system, a $3 \times 3$ grid was overlaid unto the webpage [7]. As users moved over each segment, the current location of the cursor in relation to the grid was conveyed to the user via speech. An evaluation of the system 
within a collaborative context was carried out [3]. It was found that the addition of the grid enhanced users positional awareness and led to an average of 17.3 second reduction in task completion timings. In addition, users found the concept of a grid easy to learn.

Current work focuses on the expansion of the grid concept to incorporate finer grids, such as a $27 \times 27$ grid (Figure 1). This will improve the mapping between the user's mouse movements and the movement of the cursor on screen by providing additional feedback. In addition, the page could be segmented, reducing the perceivable resolution of the screen, providing a simplified interface.

Future work will look at segmenting the page horizontally, enabling users to explore smaller sections of the page using a left to right strategy. A keyboard shortcut will be provided to enable the user to move up and down the page. This separation of horizontal and vertical exploration is hypothesized to enhance the usability of the system.

\section{EVALUATION}

Stevens et al [4] have outlined the difficulties involved in evaluating assistive technology. The second part of this research will examine techniques for evaluating assistive technology, with a focus on systems which convey spatial information. Key areas of interest will be participant profiling, training, elicitation of user's mental models, and the logging of users cursor movements.

Recruitment is an important issue with studies involving visually impaired participants. It is vital to ensure a homogeneity of variance across participant profiles. Typically blind and visually impaired users will be categorized into congenitally and adventitiously blind, blind and visually impaired, expert and novice users. However there is a wide variance within these subgroups, e.g. there are many different conditions that may render a person visually impaired, and spatial ability may vary significantly within these sub-groups. Thus the effect of a participant's spatial ability on results will be determined.

Participant training before studies is important to ensure it is the interface is being tested and not the participants' interaction with the device. Currently, training techniques and duration vary widely among studies. Research into the factors influencing adequate training will be carried out.

Another key challenge is the elicitation or externalization of user mental models. Various techniques are available - this research will focus on think-aloud, drawing and twodimensional reconstruction.

It has been recognized that adaptations are required to the think-aloud protocol to enable effective usage with visually impaired participants [7]. Such adaptations focus on the testing environment, where the auditory interface, participant and moderator dialog contribute to create a complex auditory environment. This research will look at the spatial language used by participants to describe their spatial model.

Researchers in way-finding typically encourage participants to draw the models they have generated of an area. Kitchin et al [2] describe four drawing techniques that can be used, depending on the data required.

Two-dimensional reconstruction has been used by psychologists studying the development of spatial awareness in visually impaired children. Typically, blocks are used to build up a tactile environment that reflects user's current spatial model. However this can be a slow and unfamiliar process for participants. Many visually impaired people play specially adapted board games for entertainment, e.g. chess and Sudoku. By using this familiar framework, it is hypothesized more accurate results will be gained more quickly. Bi-linear regression can then be used to analyze the differences between the original model and the model externalized by the user. Results from previous evaluations have shown that it was difficult to record and track the users' spatial movement and exploration through the system. Thus in addition to typical usability measures, such as time-on-task, task completion rate and error rate, the users' path through the system will be recorded. From this it will be possible to track areas where the user has paused or changed direction, providing an additional insight into the user's interaction with the system. It may be possible to analyze these visualizations using eye-tracking methodologies, as the mouse enables the user to "scan" the page as a sighted user would.

The evaluation framework developed will then be used to evaluate future versions of the assistive multimodal interface.

\section{CONCLUSION}

This research will provide three main contributions to HCI - i) a greater understanding of the issues visually impaired people face when accessing the Web ii) guidelines for the development for assistive multimodal systems and iii) a framework for evaluating assistive technology that conveys spatial information.

\section{ACKNOWLEDGEMENTS}

Thanks to Emma Murphy, Ravi Kuber, Graham McAllister, Wai Yu for their advice and assistance.

\section{REFERENCES}

[1] Coyne, K. and Nielsen, J. (2001). Beyond ALT text: making the web easy to use for users with disabilities. Fremont, CA: Nielsen Norman Group

[2] Kitchin, R.M. and Jacobson, R.D. (1997) Techniques to collect and analyze the cognitive map knowledge of people with visual impairments or blindness: Issues of validity, Journal of Visual Impairment and Blindness. July-August, 360-376.

[3] Kuber, R., Murphy, E., McAllister, G., Strain, P., and Yu, W. "Evaluation of an Assistive Web Interface: A Collaborative Approach”, in Press, 2007.

[4] Murphy, E., Kuber, R., McAllister, G., Strain, P., Yu, W., (2007) "An Empirical Investigation into the Difficulties Experienced by Visually Impaired Internet Users" accepted for publication Universal Access in the Information Society Journal, 2007

[5] Stevens, R., and Edwards, A. An approach to the evaluation of assistive technology. In ASSETS'96, pages 64-71. ACM Press, 1996

[6] Strain, P., McAllister, G., Murphy, E., Kuber, R. \& Yu, W., 2007: A Grid-Based Extension to an Assistive Multimodal Interface. In Proceedings of CHI '07 Extended Abstracts, San Jose, California, USA, 28 April - 3 May, 2007,

[7] Strain, P., Shaikh, A. D., and Boardman, R. 2007. Thinking but not seeing: think-aloud for non-sighted users. In CHI '07 Extended Abstracts on Human Factors in 
Computing Systems (San Jose, CA, USA, April 28 - May 03, 2007), 1851-1856.

[8] Yu, W., Kuber, R., Murphy, E., Strain, P., and McAllister, G. 2006. A novel multimodal interface for improving visually impaired people’s web accessibility. Virtual Real. 9, 2 (Jan. 2006), 133-148 